\title{
Deletion of Eqtn in mice reduces male fertility and sperm-egg adhesion
}

\author{
Chizuru Ito ${ }^{1, *}$, Kenji Yamatoya ${ }^{1,2, \dagger}$, Keiichi Yoshida ${ }^{1, \neq}$, Lisa Fujimura², Hajime Sugiyama ${ }^{3, \S,}$ \\ Akiko Suganami $^{4}$, Yutaka Tamura ${ }^{4}$, Masahiko Hatano ${ }^{5}$, Kenji Miyado ${ }^{6}$ and Kiyotaka Toshimori ${ }^{1,7, *}$ \\ ${ }^{1}$ Department of Functional Anatomy, Reproductive Biology and Medicine, Graduate School of Medicine, Chiba \\ University, Chiba, Japan, ${ }^{2}$ Biomedical Research Center, Chiba University, Chiba, Japan, ${ }^{3}$ Department of \\ Bioinformatics, Graduate School of Medicine, Chiba University, Chiba, Japan, ${ }^{4}$ Molecular Chirality Research Center, \\ Chiba University, Chiba Japan, ${ }^{5}$ Department of Biomedical Science, Graduate School of Medicine, Chiba University, \\ Chiba, Japan, ${ }^{6}$ Department of Reproductive Biology, National Center for Child Health and Development, Tokyo, \\ Japan and ${ }^{7}$ Future Medicine Research Center, Chiba University, Chiba, Japan
}

Correspondence should be addressed to K Toshimori or C Ito; Email: ktoshi@faculty.chiba-u.jp or chizuru@faculty.chiba-u.jp

*(C Ito and K Toshimori contributed equally to this work)

${ }^{\dagger}$ (K Yamatoya is now at Department of Applied Biological Science, Faculty of Science and Technology, Tokyo University of Science, Chiba, Japan)

${ }^{\ddagger}(\mathrm{K}$ Yoshida is now at Next-generation Development Center for Cancer Treatment, Osaka International Cancer Institute,

Osaka, Japan)

$\S(\mathrm{H}$ Sugiyama is now at Bio-IT R\&D Office, Fujitsu Limited, Tokyo, Japan)

\begin{abstract}
A number of sperm proteins are involved in the processes from gamete adhesion to fusion, but the underlying mechanism is still unclear. Here, we established a mouse mutant, the EQUATORIN-knockout (EQTN-KO, Eqtn ${ }^{-/-}$) mouse model and found that the

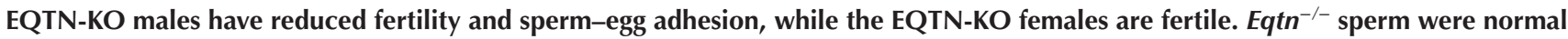
in morphology and motility. Eqtn ${ }^{-/-}-\mathrm{Tg}$ (Acr-Egfp) sperm, which were produced as the acrosome reporter by crossing Eqtn ${ }^{-/-}$with $^{-}$ $E t_{n}{ }^{+/}-\operatorname{Tg}\left(\right.$ Acr-Egfp) mice, traveled to the oviduct ampulla and penetrated the egg zona pellucida of WT females. However, Eqtn ${ }^{-/-}$ males mated with WT females showed significant reduction in both fertility and the number of sperm attached to the zona-free oocyte. Sperm IZUMO1 and egg CD9 behaved normally in Eqtn ${ }^{-/-}$sperm when they were fertilized with WT egg. Another acrosomal protein, SPESP1, behaved aberrantly in Eqtn ${ }^{-/-}$sperm during the acrosome reaction. The fertility impairment of EQTN/SPESP1-double KO males lacking Eqtn and Spesp1 (Eqtn/Spesp $1^{-/-}$) was more severe compared with that of Eqtn ${ }^{-/-}$males. Eqtn ${ }^{-/-}$-Tg (Eqtn) males, which were generated to rescue $\mathrm{Eqtn}^{-/-}$males, restored the reduced fertility.

Reproduction (2018) 156 579-590
\end{abstract}

\section{Introduction}

Fertilization is a multistep process, from sperm-egg adhesion to fusion, which is mediated by a variety of molecules in the sperm and the egg (Yanagimachi 1994, Primakoff \& Myles 2007, Evans 2012, Okabe 2013, Florman \& Fissore 2014, Toshimori \& Eddy 2014, Wassarman 2014). The sperm adhesion and fusion-related molecules must appear on the surface of the equatorial segment before the sperm reaches the surface of the egg. It is widely accepted that sperm-born IZUMO1 (Inoue et al. 2005), egg-born CD9 (Kaji et al. 2000, Le Naour et al. 2000, Miyado et al. 2000) and JUNO (Bianchi et al. 2014) are involved in gamete adhesion and/or fusion. IZUMO1 initially localized in the acrosomal membrane at the anterior acrosome before the acrosome reaction starts and relocates to the equatorial segment during the acrosome reaction (Satouh et al. 2012). Both IZUMO1 and CD9 are reported to lack features associated with fusogenic molecules (Evans 2012, Inoue et al. 2015). The egg-derived JUNOmediated dimerization of IZUMO1 facilitates sperm-egg adhesion (Inoue et al. 2015). SPESP1 (Wolkowicz et al. 2008) is reported to be one of the sperm proteins localized in the equatorial segment to maintain the integrity of the acrosomal membrane after analysis of Spesp $1^{-/-}$males (Fujihara et al. 2010); interestingly, SPESP1-deficient sperm showed an aberrant distribution and increment amounts of EQUATORIN. SPACA6, which is a predicted single-pass transmembrane protein similar to IZUMO1, 
mediates sperm-egg adhesion (Lorenzetti et al. 2014). Thus, molecules other than IZUMO1 and egg-derived CD9 could be involved in the processes from sperm-egg adhesion to fusion.

EQUATORIN is a candidate protein likely involved in fertilization (Toshimori et al. 1998) and was previously identified as an MN9 antigen specifically localized in the acrosomal membrane using the monoclonal antibody MN9 antibody (Toshimori et al. 1992). The MN9 antibody inhibited fertilization in vitro (Toshimori et al. 1998) and in vivo (Yoshinaga et al. 2001). EQUATORIN is a type 1 membrane protein, a sialoglycoprotein, with a single transmembrane domain, an N-terminus in the acrosome lumen, and a C-terminus in the cytoplasm (Yamatoya et al. 2009). We cloned the Eqtn (EQTN) gene (accession number BC089517 in the National Center for Biotechnology Information) encoding EQUATORIN (EQTN) (Yamatoya et al. 2009), also known as C9ORF11 (Ruiz et al. 2000) and AFAF (Li et al. 2006). Based on the gene and protein information (Yamatoya et al. 2009), we generated a hypothetical 3D structure of EQUATORIN (Supplementary Fig. 1A, see section on supplementary data given at the end of this article). Authentic Eqtn orthologues are conserved across mammalian species, including humans (EQTN; HUGO Gene Nomenclature Committee: 1359) (Toshimori et al. 1992, Yamatoya et al. 2009, Ito et al. 2010).

There has been one Eqtn gene-deleted mouse line, called Eqtn ${ }^{\text {tm1Gar }}$, according to the Mouse Genome Informatics. This line was originally produced by $\mathrm{Dr}$ David L Garbers and donated to the Jackson Laboratory (Stock No. 007733). A previous study examined the Eqtn $^{t m / \mathrm{Gar}}$ mice and reported that EQUATORIN-deficient males were subfertile due to sperm dysfunction in the acrosome reaction (Hao et al. 2014). We established a new mouse mutant, EQUATORIN-knockout (EQTN-KO, $\mathrm{Eqtn}^{-/-}$) mice, and investigated the role of EQUATORIN in fertilization using these mutant mice.

\section{Materials and methods}

\section{Approval of the study, ethics and animals}

This study was approved by the Biomedical Research Ethics Committee of the Graduate School of Medicine, Chiba University. All animal experiments were conducted according to the guidelines for the care and use of laboratory animals of Chiba University. C57BL/6JJmsSlc (B6) male and female mice (Japan SLC, Inc., Shizuoka, Japan, and CLEA Japan, Inc., Tokyo, Japan) were used unless otherwise stated.

\section{Generation of mutant mice}

Eqtn-deficient (-/-) mice were generated as follows. A targeting vector substituting exons $1-5$ was constructed using a pMulti-ND-1.0 targeting vector (provided by Drs J Takeda and T ljiri) containing the Neo resistance gene cassette $\left(\right.$ neo $\left.^{r}\right)$ as a positive selection marker and diphtheria toxin A chain (DT) as a negative selection marker. A 6.1-kbp fragment long arm and a 2.1-kbp fragment short arm were obtained by PCR amplification using a BAC clone (RPCI-23 MM BAC CLONE: 135A21, Invitrogen, Carlsbad, CA) as a template. The PCR primers were (5'-3'): GAGCTCGAGCTCCGCCACACAATGGAGTCTTTGTC and GAGCTCGAGCTCCTCCAGGGAAATGTCCAGAGC for the long arm and CTGCAGAGGTTGCAGTCTAGAGTG and AGTGCAACACCCTGAGATGACG for the short arm. The fragment for the long arm was cloned into a pTA2 vector (TOYOBO Co., LTD. Osaka, Japan), digested using Xhol, and inserted into the Xhol site of the pMulti-ND 1.0 vector. The fragment for the short arm was digested with Xbal, inserted in pMulti-ND 1.0 vector, redigested using Mlul and inserted into the pMulti-ND 1.0 vector containing the long arm fragment. R1 embryonic stem cells derived from 129/Sv were electroporated with Pmel-digested linearized DNA, and 263 clones were examined using PCR and Southern blot analyses. Screening primers were $\left(5^{\prime}-3^{\prime}\right)$ TGACCGCTTCCTCGTGCTTT and GCCTATGGTTCATGACACTG for the short arm and CACATGGCTCTGGCTTCTCCAAAG and CCTACCCGCTTCCATTGCTCA for the long arm. Both ends of the Eqtn-deleted allele in the embryonic stem cells were confirmed by Southern blot analysis. The $5^{\prime}$ and $3^{\prime}$ probes for Southern blot analysis were amplified from genomic DNA using the following primers: 5'-CACACCTCAGTACTACAGAATGGG-3'

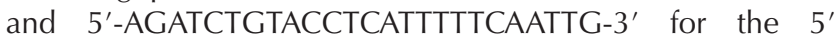
probe and 5'-CCTATTCAAACCACCATAGGAAAT-3' and 5'-ACTTTAATGATTGTACCTGTGTC-3' for the $3^{\prime}$ probe. The probes were radioisotope-labeled using the Megaprime DNA Labeling System (GE Healthcare, Buckinghamshire, UK). Xhol-digested genomic DNA from embryonic stem cells carrying the deleted allele was hybridized with $5^{\prime}$ and $3^{\prime}$ probes. Homologous recombination was detected in 19 clones. Using the aggregation method, one independent, targeted embryonic stem cell clone was used to generate chimeric mice. Tail DNA from agouti pups obtained from mating with C57BL/6 mice was analyzed using PCR. The genotyping primers were $\left(5^{\prime}-3^{\prime}\right)$ : AAACAAAACCCCCAAATTCC (forward), GCTTGGCTGGACGTAAACTC (reverse) and GGCAGGAGTGTCCATTTCAT (reverse). The 726 bp and 465 bp fragments represent wild-type $(\mathrm{WT} ;+/+)$ and homozygous $(-/-)$ mutants, respectively. Heterozygous $(+/-)$ mutant mice were maintained by crossing with $\mathrm{C} 57 \mathrm{BL} / 6 \mathrm{JJ} \mathrm{msSlc}$ mice, and Eqtn-homozygous mutants $(-/-)$ were generated by intercrossing heterozygous mutant (+/-) mice. The mutant mice were back-crossed more than ten times to make the congenic line. Rescued mice, Eqtn ${ }^{-/-}$-transgenic (Tg) (Eqtn) mice (termed as $E q t^{-/-}$- Tg (Eqtn) mice), were generated as follows. pCMEqtn was made by deleting enhanced green fluorescent protein $(E G F P)$ from the pCM-Eqtn-Egfp vector (Ito et al. 2013) by PCR using 5'-TAAGGATCCCCCGGGCTGCAGGAATTC-3' and 5'-ATTACTCGGTGATCCTTCCTTGTTCAAC-3' primers. The transgene excised from pCM-Eqtn using Scal, Sall and EcoRV was then purified, injected into Eqtn ${ }^{-/-}$-fertilized eggs, and they were then transferred to the oviducts of pseudopregnant mice. The transgene transmittance was examined by PCR with 5'-CTGATGACACTGGTTCTGCTAATCC-3' and 5'-ATTACTCGGTGATCCTTCCTTGTTC-3' primers. The generated males (rescue mice) were healthy and showed 
the expression of inserted Eqtn as confirmed by indirect immunofluorescence (IIF).

The acrosome reporter, Acrosin/Egfp transgene (Acr-Egfp) mice (Nakanishi et al. 1999) provided by Dr Masaru Okabe (Osaka University, Osaka, Japan) and termed as Eqtn ${ }^{+/+}$ $\operatorname{Tg}($ Acr-Egfp $)$ mice, was maintained in our laboratory and used as controls to monitor the sperm journey in the oviduct


$\operatorname{Tg}($ Acr-Egfp $)$ mice were generated by crossing Eqtn ${ }^{-/-}$with Eqtn ${ }^{+/+}-\mathrm{Tg}($ Acr-Egfp $) \quad$ mice. Cd9 ${ }^{-/-}-\mathrm{Tg}(\mathrm{Cd}$ 9-Egfp $)$ mice (Miyado et al. 2008) were maintained in our laboratory and used to report intact egg-derived CD9. Eqtn/Spesp $1^{-1-}$ mice were generated as follows. B6;129S2-Spesp1<tm1Osb $>$ (RBRC04714) mice (Spesp $1^{+/}$) were provided by the RIKEN BioResource Center through the National Bio-Resource Project of MEXT, Japan, which was previously reported (Fujihara et al. 2010). Using Spesp $1^{+/-}$mice (four males and four females), we generated Spesp $1^{-/-}$mice. We generated Eqtn/Spesp $1^{-/-}$ mice by crossing Spesp $1^{-/-}$mice with $E q t n^{-/-}$mice for this study. Genotyping for Eqtn- and Spesp1-null mutations was performed by PCR of tail DNA using the following sets of primers: Eqtn1, 5'-AAACAAAACCCCCAAATTCC-3', 5'-GGCAGGAGTGTCCATTTCAT-3'

5'-GCTTGGCTGGACGTAAACTC-3';

and

$5^{\prime}$ - CTTTTCTCTCAGCTCTGATGGTACACGCTT- ${ }^{\prime}$, 5' - GCCTTCTATCGCCTTCTTGACGAGTTCTTC-3' and 5'-TCCGGTGAGCCAGTGTGCGTCTAC-3'.

\section{Molecular modeling of EQUATORIN}

The 3D structure of the murine EQTN long form (Supplementary Fig. 1A) was constructed using the Molecular Operating Environment software (version 2008; CCG, Montreal, Canada). Molecular modeling of the $\mathrm{N}$-terminus or luminal (21-184) and the C-terminus or cytoplasmic (207-337) domains of murine EQTN were constructed using the homology modeling module in the Molecular Operating Environment software based on the Brookhaven Protein Databank $1 \mathrm{NI} 5$ and 3B5H, respectively. In addition, the transmembrane (185-206) domain was constructed using the building module in the Molecular Operating Environment software. After joining these domains, molecular mechanics calculations were performed to obtain the local minimum energy structure of the murine EQTN long form. Then, lipid bilayer membranes were constructed using the structure-building tools of the VMD (http://www.ks.uiuc. edu/Research/vmd/). Finally, the murine EQTN long form was inserted into the lipid bilayer for the molecular dynamics simulations using AMBER 9 (http://ambermd.org/). The 3D structures of the complexes were displayed using MolFeat (version 4; FiatLux).

\section{Antibodies and chemicals}

The primary antibodies used were mouse monoclonal antibody MN9 ( $\operatorname{lgG}_{2 \mathrm{a}}$ ) (Toshimori et al. 1992), rabbit polyclonal antibody EQ70-83 (Yamatoya et al. 2009), guinea pig polyclonal antiSPACA1 (American Research Products, Inc., Waltham, MA, USA), mouse monoclonal anti-ADAM3 (clone 7c1.2; mouse $\operatorname{IgG}_{1} ;$ Merck), mouse monoclonal anti- $\beta$-TUBULIN (clone tub
2.1; Sigma-Aldrich), rabbit polyclonal anti-SNAP25 (Abcam), rat monoclonal anti-mouse JUNO/FOLR4 antibody (clone TH6; BioLegend, Inc., San Diego, CA, USA), rat monoclonal anti-mouse CD9 antibody (BD Biosciences San Jose, CA, USA). Rabbit polyclonal anti-SPESP1 and rat monoclonal antiIZUMO1 antibodies were provided by Drs Okabe and Ikawa from the Osaka University. The secondary antibodies used for IIF were Alexa Fluor 488 goat anti-mouse $\operatorname{lgG} 2_{a^{\prime}}$ anti-rabbit $\lg \mathrm{G}$, anti-guinea pig IgG, anti-rat $\lg \mathrm{G}$, donkey anti-goat IgG, and Alexa Fluor 555 goat anti-mouse IgG and donkey antirat $\mathrm{IgG}$ at $0.5 \mu \mathrm{g} / \mathrm{mL}$ (Molecular Probes). HRP-conjugated sheep anti-mouse IgG antibodies and donkey anti-rabbit IgG antibody (GE Healthcare), goat anti-rat IgG antibody (Jackson Immunoresearch) and anti-guinea pig IgG (Chemicon International, Temecula, CA, USA) were used for western blotting. Hoechst 33342 was used at $0.3 \mu \mathrm{g} / \mathrm{mL}$ for imaging the nuclei.

\section{Western blotting}

SDS-PAGE and immunoblotting were performed as described previously (Yamatoya et al. 2009). In brief, the samples were extracted with SDS sample buffer (50 mM Tris- $\mathrm{HCl}(\mathrm{pH} 6.8)$, $2 \%$ SDS, $100 \mathrm{mM}$ dithiothreitol (DTT), 10\% (v/v) glycerol, and $0.002 \%(\mathrm{w} / \mathrm{v})$ bromophenol blue). The lysates were heated for $10 \mathrm{~min}$ at $98^{\circ} \mathrm{C}$ and were centrifuged at $16,000 \mathrm{~g}$ for $10 \mathrm{~min}$ to remove insoluble material. The lysates were then separated by SDS-PAGE and were blotted onto a PVDF membrane (PVDF, Millipore). Western blot analysis was performed according to a standard protocol using TBS-T (20 mM Tris- $\mathrm{HCl}(\mathrm{pH} \mathrm{7.6),}$ $137 \mathrm{mM} \mathrm{NaCl}$ and $0.1 \%(\mathrm{w} / \mathrm{v})$ Tween 20$)$ containing 5\% (w/v) skimmed milk as a blocking solution and for antibody dilution. The antibody concentrations used for the western blot analysis were $0.1 \mu \mathrm{g} / \mathrm{mL}$ for $\mathrm{MN9}, 0.3 \mu \mathrm{g} / \mathrm{mL}$ for EQ70-83, 1:4000 dilution for SPACA1, 1:4000 dilution for SPESP1, $0.1 \mu \mathrm{g} / \mathrm{mL}$ for SNAP25, $0.3 \mu \mathrm{g} / \mathrm{mL}$ for ADAM3, 1:4000 dilution for IZUMO1 and $0.5 \mu \mathrm{g} / \mathrm{mL}$ for $\beta$-TUBULIN antibodies and $1: 20,000$ dilution for HRP-conjugated secondary antibodies. The blots were developed using ECL prime western blotting Detection Reagents (GE Healthcare) and were exposed to X-ray film or alternatively they were developed with the Clarity western ECL substrate (Bio-Rad Laboratories, Inc.) and visualized with a ChemiDoc XRS Plus system (Bio-Rad).

\section{Analyses of fertility, sperm-fertilizing ability and imaging system}

We performed fertility tests by counting the number of fetuses and vestiges as well as measuring the body weight of fetuses in uteri of $\mathrm{Eqtn}^{+/+}$females at 17 days postplug formation (PPF) because the quantification of newborns was unreliable due to loss by cannibalism. In the rescue experiment, we also counted the number of pups obtained by natural mating. The fertility test was performed by mating males with females in their natural estrous cycles. The mating was confirmed by detecting vaginal copulation plug formation at $9 \mathrm{am}$ each morning. Next, we performed in vivo fertilization assays to determine the rate of 2-cell and pronucleus formation. For this purpose, we observed the mating behavior with our 
own eyes and by video recording to precisely determine the copulation time. We killed the females with copulation plugs at $24 \mathrm{~h}$ PPF for two-cell formation and at $10 \mathrm{~h}$ and $5 \mathrm{~h}$ PPF for pronucleus formation. Hormonally treated females were used for these experiments (Yoshinaga et al. 2001). For two-cell and pronucleus formation assays, we counted the number of zygotes after recovering samples from the female oviducts. For pronucleus formation assays, we counted the number of zygotes with sperm heads and pronuclei by staining them with $1 \mu \mathrm{g} / \mathrm{mL}$ Hoechst 33342 (Sigma-Aldrich). The fertility rate was calculated as follows: the total number of fertilized eggs/ total number of eggs counted. Females with many (>20\%) unusual eggs (dead, fragmented and immature germinal vesicle stage eggs) were excluded from the statistical analyses. Observations were generally made under an Olympus IX71 inverted microscope (Olympus) or a BX50 (Olympus) microscope equipped with UPlanFLN 20x and UPlanApo $40 \times$ dry objective lenses (Olympus). The imaging systems were composed of appropriate filters for fluorescence, and a RETIGA Exi FAST 1394 CCD (Charge-Coupled Device) camera (Qimaging, Surrey, BC, Canada). The SlideBook 4/5 software (Intelligent Imaging Innovations, London, UK) controlled the data acquisition and storage, including Z-axis analysis and 3D reconstruction. Photographs were taken under an Axiovert 135 inverted microscope (Zeiss) equipped with a Leica MC170 HD camera (Leica Microsystems).

\section{Binding assay of sperm to zona-free oocyte (gamete binding assay) and IVF}

\section{Preparation of spermatozoa}

Sperm and oocytes were collected as reported previously (Toshimori et al. 1998) with some modifications for this study. In brief, sperm were collected from the cauda epididymides of Eqtn ${ }^{+/+}$and Eqtn ${ }^{-/-}$males (10-12 weeks of age). Dense sperm masses were squeezed out of the epididymis of B6D2F1 males by pricking the wall with a $28 \mathrm{G}$ injection needle, and sperm were allowed to disperse sperm in a tube containing $500 \mu \mathrm{L}$ TYH (a modified Kreb's-Ringer bicarbonate solution) (Toyoda et al. 1971) medium. The sperm were then incubated for $3 \mathrm{~h}$ at $37^{\circ} \mathrm{C}$ in $5 \% \mathrm{CO}_{2}$ in air to induce capacitation.

\section{Gamete-binding assay}

This assay was performed basically according to the methods reported previously (Wang et al. 2017). Modifications were made for this study as follows. Oocytes were collected from female mice ( 7 weeks of age) that were stimulated with 5IU of PMSG (ASKA Animal Health Co., Ltd, Tokyo, Japan) followed by $48 \mathrm{~h}$ later by hCG (ASKA) $48 \mathrm{~h}$ later. Cumulusoocyte complexes were collected 15-16h post hCG and washed in TYH medium. The cumulus cells were removed by treatment with $0.5 \mathrm{mg} / \mathrm{mL}$ hyaluronidase (Sigma-Aldrich) in TYH medium. After brief washing in TYH medium, the eggs were consecutively placed in three drops of acidic Tyrode's solution ( $\mathrm{pH} 2.5$, Sigma-Aldrich) for 5-10s to remove the zona, and the zona-free oocytes; they were then washed in three drops of TYH medium and incubated in a $\mathrm{CO}_{2}$ incubator for $1 \mathrm{~h}$ for recovery. The oocytes were then inseminated with sperm in an IVF dish containing TYH medium, at a final concentration of $1 \times 10^{4}$ sperm/dish. After $1 \mathrm{~h}$ of coincubation in a $5 \% \mathrm{CO}_{2}$ incubator at $37^{\circ} \mathrm{C}$, the oocytes were transferred to a drop $(5 \mu \mathrm{L})$ of Hoechst 33342 in TYH medium in $35-\mathrm{mm}$ glass bottom dishes. Immediately, these dishes were put on the automated stage top in the incubation chamber supplemented with $5 \% \mathrm{CO}_{2}$ at $37^{\circ} \mathrm{C}$ (INU system, Tokai Hit, Shizuoka, Japan) to start live cell imaging by the Olympus IX71 inverted fluorescence microscope equipped with UPlanApo $20 \times$ NA objective lens (Olympus) and RETIGA Exi FAST 1394 CCD camera controlled by SlideBook $4 / 5$ software as described earlier. After approximately $10 \mathrm{~min}$, the tail movement naturally ceased due to inactivation of the nuclear activity by Hoechst staining, and we then acquired images of all eggs examined as Z-stack images of fluorescence and DIC at $0.5 \mu \mathrm{m}$ intervals. By comparing the different focal planes (Z-axis analysis), sperm nuclei that were sharply and strongly stained with a dot shape (approximately $2-5 \mu \mathrm{m}$ ) could be readily differentiated from oocyte chromosomes (MII) that were found to be of much bigger shapes with characteristic MII shapes (approximately $10-15 \mu \mathrm{m}$ in width or diameter). Polar bodies were differentiated from sperm nuclei due to their unique localization in the perivitelline space (PVS) with corresponding nuclear fluorescence. Using this sperm concentration, it was not difficult to count the number of sperm that bound the egg. The obtained data were statistically analyzed.

\section{Live cell imaging with Eqtn ${ }^{-/-}$-Tg(Acr-Egfp)}

To examine the journey of Eqtn ${ }^{-/}$sperm in the oviduct from the uterus to the ampulla (eggs), Eqtn ${ }^{-1-}$-Tg(Acr-Egfp) males were mated with Eqtn ${ }^{+/+}$females that were artificially ovulated. Females with copulation plugs were sacrificed at $5 \mathrm{~h}$ PPF. After the uterus and oviduct were removed from the plug-forming females, we placed the oviducts with uteri into in vitro fertilization (IVF) dishes containing TYH medium and stored them in a $5 \% \mathrm{CO}_{2}$ incubator at $37^{\circ} \mathrm{C}$ until use. The sperm journey in the oviduct was monitored by following the green sperm (GFP-green acrosome) through the extended oviduct wall and was visualized under a Leica M165 FC fluorescence stereomicroscope equipped with a PLANAPO $\times 1.0$ lens and a DFC 310 FX camera (Leica Microsystem). We compared the images with those of control Eqtn ${ }^{+/+}-\mathrm{Tg}(\mathrm{Acr}$ Egfp) sperm. Live cell imaging was recorded for up to $7 \mathrm{~h}$ or until the fluorescence faded out or the sperm motility ceased. Recording occurred at varying interval times depending on the purposes of the experiments; e.g., $1.5 \mathrm{~ms}$ on average for live imaging with the Olympus IX71 (Olympus) inverted fluorescence microscope described above and equipped with an automated stage top $5 \% \mathrm{CO}_{2}$ incubator at $37^{\circ} \mathrm{C}$ (INU system, Tokai Hit, Shizuoka, Japan).

\section{IIF microscopy and assessment of the acrosome reaction}

Cauda epididymal sperm (cauda sperm) were collected from Eqtn $^{+/+}$and Eqtn ${ }^{-/-}$males as described above. Sperm for IIF examination except for live imaging were fixed for $10 \mathrm{~min}$ with $4 \%$ paraformaldehyde after rinsed with phosphate 
A

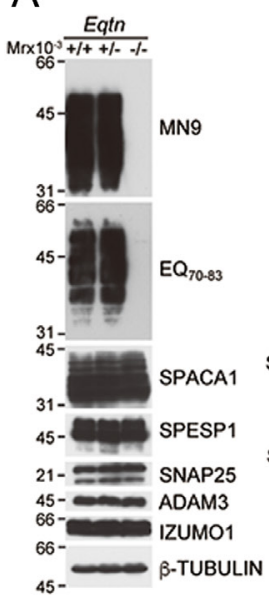

B

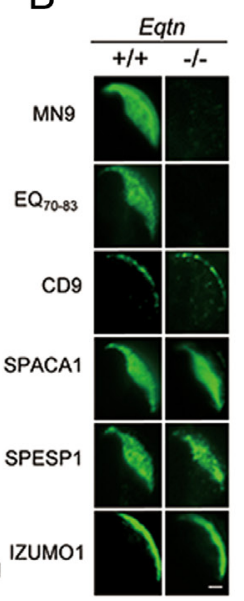

C

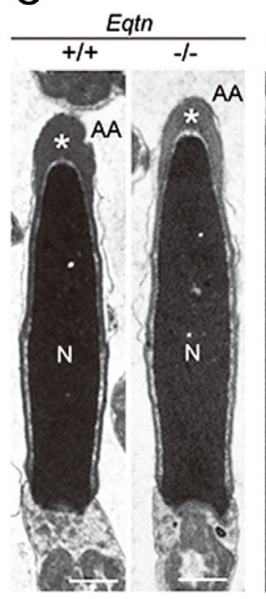

D

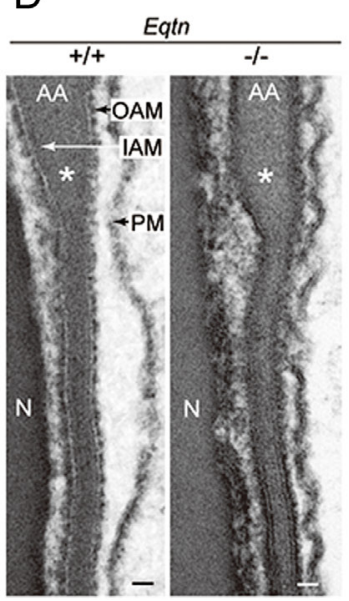

E

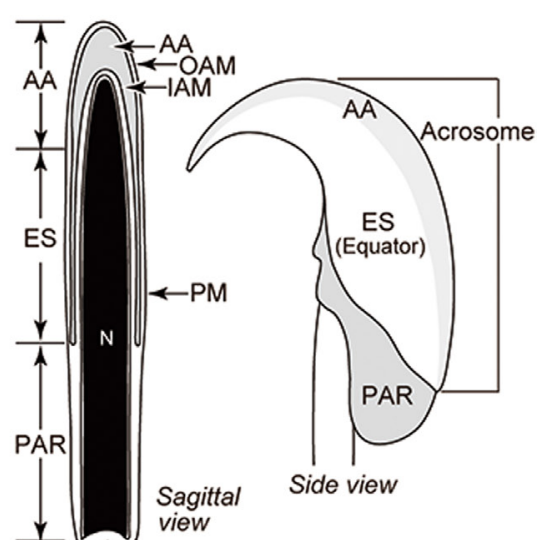

Figure 1 Features of Eqtn deletion and Eqtn ${ }^{-/-}$sperm morphology. (A and B) Eqtn deletion and presence of major fertilization-related proteins known so far in Eqtn ${ }^{-/-}$sperm (-/-). +/+; Eqtn ${ }^{+/+}$sperm. (A) Western blotting. $\beta$-TUBULIN is the internal control. Approximately $10^{4}$ sperm were loaded per lane. Three males each of Eqtn ${ }^{+/+}(+/+)$, Eqtn ${ }^{+/-}(+/-)$and Eqtn ${ }^{-/-}(-/-)$were examined and duplicated. (B) IIF with antibodies for anti-acrosomal proteins. Cauda sperm after $4 \%$ paraformaldehyde fixation and $0.1 \%$ Triton-X treatment to permeabilize the membrane. More than 50 sperm were examined for each staining. $N=3$ (3 males). Scale bar, $1 \mu \mathrm{m}$. (C and D) Transmission electron microscopy images showing normal morphology of the head region in Eqtn ${ }^{-/-}$sperm (-/-). +/+; Eqtn ${ }^{+/+}$sperm. More than 50 sperm were analyzed for Eqtn ${ }^{+/+}(+/+)$and Eqtn $^{-/-}(-/-) . N=3$ (3 males). (C) Low magnification showing normal arrangement of head components such as the acrosomal matrix $(*)$. Scale bar, $0.5 \mu \mathrm{m}$. (D) Higher magnification showing a normal arrangement of components localized at the equatorial segment region such as the inner acrosomal membrane, outer acrosomal membrane and matrix substances $\left(^{*}\right)$. Scale bar, $20 \mathrm{~nm}$. (E) Schematic diagram showing major regions and elements of the mouse sperm head that were mainly addressed in this study. AA, anterior acrosome; ES, equatorial segment; $\mathrm{N}$, sperm nucleus; PAR, postacrosomal region; PM, plasma membrane.

buffered saline and were briefly treated with $0.1 \%$ Triton-X 100 to permeabilize the membranes immediately after being removed from the epididymis. Sperm for examination of the acrosome reaction or the acrosome-reacted sperm were not treated with Triton-X 100. After washing in phosphate buffered saline, sperm were incubated with primary antibodies against MN9, EQ70-83, SPACA1, SPESP1 and IZUMO1 at $4{ }^{\circ} \mathrm{C}$ overnight and were incubated with secondary antibodies at room temperature for $30 \mathrm{~min}$. These samples were analyzed with a conventional, high-resolution (BX 50, Olympus), epifluorescence microscope (IX71, Olympus) as previously reported (Ito et al. 2015). IIF was performed to identify the proteins of interest, such as EQUATORIN and IZUMO1, under in vivo experiments by mating males with females. To assess the acrosome reaction, or to see the immunostaining difference of SPESP1 during the acrosome reaction, sperm were incubated in TYH medium in a humidified atmosphere of $5 \%(\mathrm{v} / \mathrm{v}) \mathrm{CO}_{2}$ in air at $37^{\circ} \mathrm{C}$ for $3 \mathrm{~h}$ to induce a spontaneous acrosome reaction, and they were then treated in $4 \%$ paraformaldehyde for $10 \mathrm{~min}$ to stop the progression of the acrosome reaction. The stage of acrosome reaction was determined based on the IZUMO1 staining pattern. Images were taken as described above.

\section{Assessment of sperm motility and morphology}

Fresh sperm were collected from cauda epididymides. After washing with TYH medium, sperm were examined with a differential interference contrast microscope with an UPlanApo $100 \times$ NA oil objective lens (Olympus). Images were captured with a CCD camera controlled by the SlideBook 5.5 software (Intelligent Imaging Innovations) in a BX50 light microscope
(Olympus). After incubation in a humidified atmosphere with $5 \%(\mathrm{v} / \mathrm{v}) \mathrm{CO}_{2}$ in air at $37^{\circ} \mathrm{C}$ for $30 \mathrm{~min}$, sperm motility and other characteristic parameters were quantitatively analyzed with an automated Sperm Motility Analysis System (SMAS; DITECT Co. Ltd, Tokyo, Japan). Transmission electron microscopy was performed to analyze the morphological details of sperm and spermatogenesis. Testes and cauda epididymides were fixed with $2.5 \%$ glutaraldehyde in $0.1 \mathrm{M}$ phosphate buffer, postfixed with $1 \% \mathrm{OsO}_{4}$, dehydrated, and embedded in Epon 812. Onemicrometer sections were cut on an ultramicrotome (Ultracut E; Reichert-Jung, Vienna, Austria) and were stained with $1 \%$ toluidine blue for light microscopy. Ultrathin sections were stained with uranyl acetate and lead citrate for transmission electron microscopy (1200EX, JEOL, Tokyo, Japan), as previously reported (Ito et al. 2015).

\section{Flow cytometry for the acrosome reaction}

Cauda sperm from Eqtn ${ }^{-/-} \operatorname{Tg}($ Acr-Egfp) mice and control Eqtn ${ }^{+/+}-\mathrm{Tg}($ Acr-Egfp) mice were suspended in $1 \mathrm{~mL}$ of TYH medium and were centrifuged at $163.5 \mathrm{~g}$ for $15 \mathrm{~min}$ to remove the epididymal fluid. The sperm were suspended in fresh TYH medium containing $10 \mu \mathrm{M}$ calcium ionophore A23187 (SigmaAldrich) to control the start of the acrosome reaction and were centrifuged at $163.5 \mathrm{~g}$ for $10 \mathrm{~min}$ to remove the $\mathrm{A} 23187$. The sperm were then suspended in fresh $\mathrm{TYH}$ medium, incubated for $0,15,30$ or $60 \mathrm{~min}$ at $37^{\circ} \mathrm{C}$ in a $5 \% \mathrm{CO}_{2}$ incubator, and fixed in $4 \%$ paraformaldehyde for $10 \mathrm{~min}$ at room temperature to stop the acrosome reaction. Paraformaldehyde cross-linking was quenched by washing with TYH medium. Sperm were not treated with Triton-X 100. We performed flow cytometric 
analyses with a FACSCanto II (BD Biosciences) and the data were analyzed using the Flowjo software (Tree Star, Inc., Ashland, OR).

\section{Statistical analysis}

All data are shown as the mean \pm standard error of the mean (S.E.M.). For statistical analysis, we performed a TukeyKramer test for reduction of fertility, Mann-Whitney's $U$ test for reduction in the body weight of fetuses, 2-cell formation rate at $24 \mathrm{~h}$ PPF, pronucleus (PN) formation rate at $10 \mathrm{~h}$ and increment in the number of MII eggs with Eqtn-/- sperm in the PVS (PVS sperm) at $10 \mathrm{~h}$ and $5 \mathrm{~h}$ PPF, Student's $t$-test for reduction of $\mathrm{PN}$ formation rate at $5 \mathrm{HPPF}$ and Supplementary Fig. 2A (testis and epididymis), Welch's t-test for binding of sperm to zona-free oocyte, Steel-Dwass test for comparing average number of fetuses/pups in EQTN-Ko and EQTN/ SPESP1-double KO with wild-type, and Steel's test for rescue of Eqtn-/- male fertilityreduction by Eqtn-/--Tg(Eqtn) males after mating with wild-type females using the add-in Statcel 3 software (OMS, Ltd, Tokyo, Japan) for Microsoft Excel. Probability $(P)$ values were regarded as statistically significant at $P<0.05$. ${ }^{*} P<0.05 ;{ }^{*} P<0.01$. We collected and processed the data in a randomized manner.

\section{Results}

\section{Eqtn $^{-/-}$sperm are normal in morphology and motility}

Exons 1-5 were deleted in the newly generated $E_{\text {En }}{ }^{-/}$mouse line by targeted disruption of the Eqtn gene (Supplementary Fig. 1B). Gene disruption in the embryonic stem cells and germline transmission were confirmed by PCR and Southern blot genotyping (Supplementary Fig. 1C). The offspring production from intercrossing Eqtn ${ }^{+/-}$mice followed a Mendelian inheritance ratio (Supplementary Fig. 1D). EQUATORIN was deleted in Eqtn ${ }^{-/}$sperm, but major proteins, including SPACA1, SPESP1, SNAP25, ADAM3, IZUMO1 and sperm CD9, were present at normal levels, as shown by western blotting or immunofluorescence of epididymal sperm (Fig. 1A and B). Eqtn ${ }^{-/-}$sperm were normal in phenotypes by light and electron microscopy, with no apparent anomalies in the sperm head structures, including the acrosome (Fig. 1C and D and Supplementary Fig. 2A). Eqtn ${ }^{-/-}$males showed no developmental abnormalities and were normal with respect to sexual behavior, features of reproductive organs, spermatogenesis, sperm morphology and motility (Supplementary Fig. 2A, B and C). In addition, Eqtn ${ }^{-1-}$ $\mathrm{Tg}(A c r-E g f p)$ sperm showed a normal migration pattern during the journey to the ovulated eggs in the oviduct ampulla, similar to that of control Eqtn ${ }^{+/+}-\mathrm{Tg}($ Acr-Egfp $)$ sperm (Supplementary Fig. 2C). Eqtn ${ }^{-1-}$ Tg(Acr-Egfp) sperm showed normal acrosome reaction progression patterns compared to control Eqtn ${ }^{+/}-\mathrm{Tg}(\text { Acr-Egfp })_{1}$ sperm, as shown by flow cytometry (Supplementary Fig. 2D). Thus, Eqtn ${ }^{-/}$males appeared normal, but we hypothesized that Eqtn ${ }^{-/-}$sperm would be functionally defective because the anti- EQUATORIN antibody MN9 inhibited fertilization.

\section{EQUATORIN-deficiency reduces male fertility and sperm-egg adhesion}

To examine the fertility of $\mathrm{Eqtn}^{-/-}$males, in vivo fertility assays were performed by mating WT $\left(E q t n^{+/+}\right), E q t n^{+/-}$ and $E_{t n^{-1}}$ males with WT females. As a result, both the average number (Fig. 2A) and the body weight (Fig. 2B) of fetuses at 17 days PPF were significantly lower in Eqtn $^{-/-}$males compared to Eqtn ${ }^{+/+}$and Eqtn ${ }^{+/-}$males. The two-cell formation rate at $24 \mathrm{~h}$ PPF (Fig. 2C) and the pronucleus formation rate at $10 \mathrm{~h}$ and $5 \mathrm{~h}$ PPF (Fig. 2D) were significantly lower in Eqtn ${ }^{-/-}$sperm than in Eqtn ${ }^{+/+}$ sperm. In the fertility assay (Fig. 2E and Supplementary Fig. 3), almost similar number of $\operatorname{Tg}($ Acr-EGFP) sperm appeared to travel in the oviduct of WT females which were mated with Eqtn ${ }^{+/+}$and Eqtn ${ }^{-/-}$males (Supplementary Fig. 2C), and the sperm normally penetrated the zona pellucida, but the sperm accumulated in the PVS (PVS sperm) were more frequently found in $\mathrm{Eqtn}^{-/-}$males than in $\mathrm{Eqtn}^{+/+}$males. The number of MII eggs that had redundant PVS sperm at $10 \mathrm{~h}$ and $5 \mathrm{~h}$ PPF increased significantly in Eqtn $^{-/-}$males than in Eqtn ${ }^{+/+}$males (Fig. 2E). The time-lapse imaging frequently showed $E_{\text {En }}{ }^{-1-}$ sperm vigorously moving in PVS, suggesting that the fertility defect was attributed to sperm-egg adhesion (Fig. 2F and Video 1). The gamete binding assay using activated sperm and zona-free oocyte demonstrated that the number of sperm attached to oocytes was significantly lower in Eqtn ${ }^{-/-}$than in WT males (Fig. 2G and Supplementary Fig. 4).

\section{Video 1}

Eqtn -/- sperm in PVS. Videos were taken from three Eqtn-/- males. The video (http://movie-usa. glencoesoftware.com/video/10.1530/REP-18-0394/ video-1) from the online version of the article is available at https://doi.org/10.1530/REP-18-0394.

\section{IZUMO1 and CD9 are present in Eqtn ${ }^{-/-}$sperm that reached the PVS}

To determine whether IZUMO1 is present or not in $E_{\text {En }}{ }^{-/}$sperm that reached the PVS, we examined eggs obtained from $\mathrm{Eqtn}^{+/+}$females after mating with Eqtn ${ }^{-/-}$males. IZUMO1 was present in Eqtn ${ }^{-/-}$ sperm that were accumulated in the PVS of MII eggs (Fig. 3A). Similarly, to determine whether CD9 is present or not in $\mathrm{Eqtn}^{-/-}$sperm that reached the PVS, we examined eggs obtained from $\mathrm{Cd} 9^{-/-} \mathrm{Tg}(\mathrm{Cd} 9$ Egfp) females after mating with Eqtn ${ }^{-/-}$males. CD9 was present in Eqtn ${ }^{-/-}$sperm that were accumulated in the PVS of MII eggs (Fig. 3B). The PVS of $\mathrm{Cd}^{-/-}$$\mathrm{Tg}(\mathrm{Cd}$ 9-Egfp) eggs was filled with CD9 (Fig. 3B) and JUNO (Supplementary Fig. 5). 
A

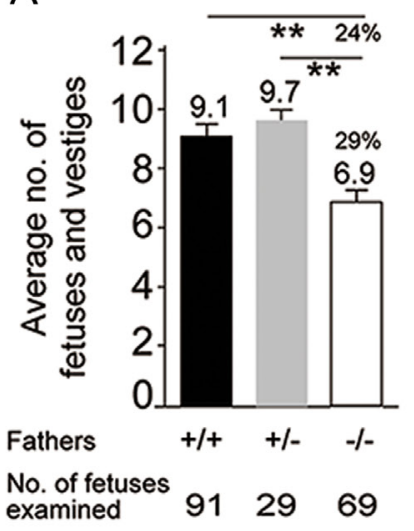

No. of matings $10 \quad 3 \quad 10$
B

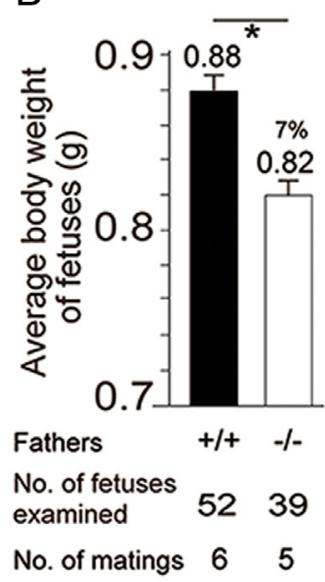

C

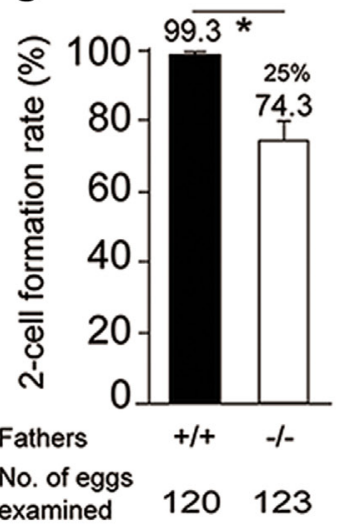

No. of matings $6 \quad 6$

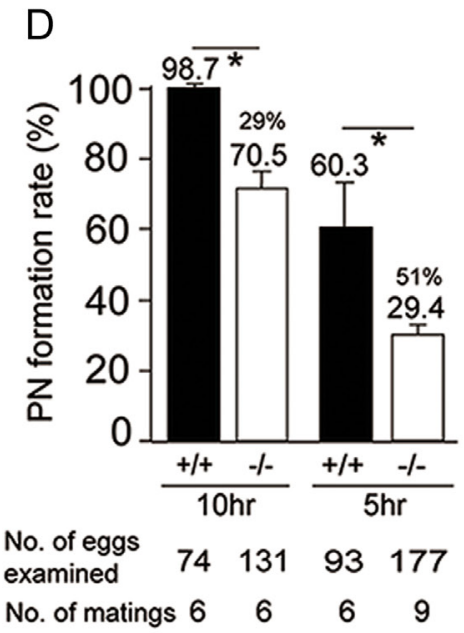

Ea

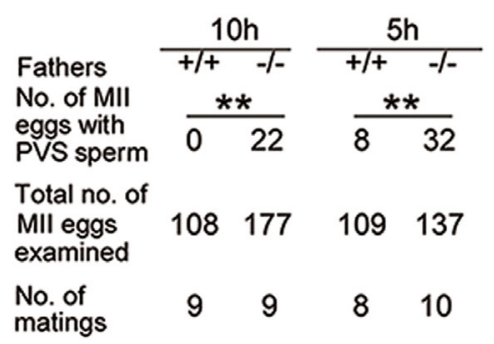

F

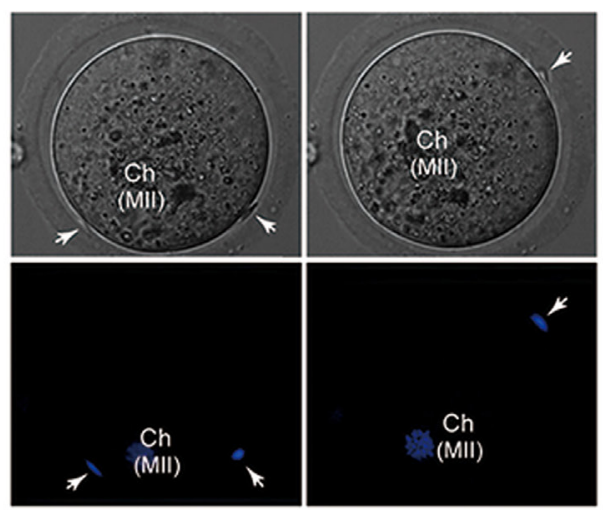

G

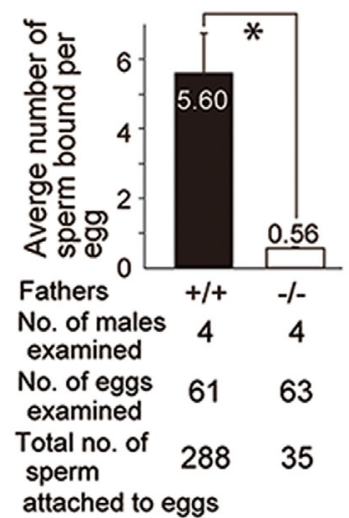

b

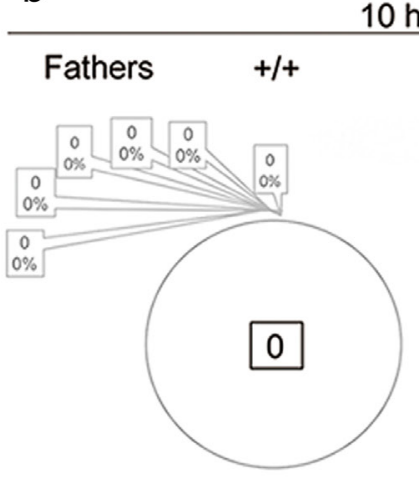
$10 \mathrm{hrs}$

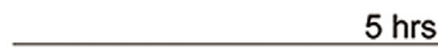

$5 \mathrm{hrs}$

$+/+$ $-1-$


Figure 2 Failures caused by Eqtn ${ }^{-/}$males (sperm) after mating with WT females. (A and B) 17 days postplug formation (PPF). (A) Reduction of fertility. (B) Reduction of the body weight of fetuses. (C) Reduction of 2-cell formation rate at $24 \mathrm{~h}$ PPF. (D) Reduction of pronucleus (PN) formation rate at $10 \mathrm{~h}$ and $5 \mathrm{~h}$ PPF. (E) a: Increment in the number of MIl eggs with Eqtn ${ }^{-1-}$ sperm in the PVS (PVS sperm) at $10 \mathrm{~h}$ and $5 \mathrm{~h}$ PPF. b: Details of a (pie chart; \%). The pie charts show the percentage (\%) of MIl eggs containing $1 \sim>6 \mathrm{Eqtn}^{-/-}$sperm that are present in the PVS. (F) Still frame showing two $\mathrm{Eqtn}^{-/-}$sperm in PVS (Video 1) at $5 \mathrm{~h}$ PPF. Differential interference contrast image. Arrows indicate sperm heads. This egg chromosome (Ch) was MII. Blue (Hoechst) for sperm nuclei and egg chromosome (MII). Similar images were taken from 12 Eqtn ${ }^{-/-}$males; videos were taken from three $\mathrm{Eqtn}^{-/-}$males. Scale bar, $10 \mu \mathrm{m}$. (G) Binding of sperm to zona-free oocyte. The average number of sperm bound per eggs is significantly lower in Eqtn ${ }^{-/}$cases (-/-) than in WT cases (+/+). Bars in the graph show S.E.M. (standard error of the mean). Detailed data are shown in Supplementary Fig. $4 .{ }^{*} P<0.05$; ${ }^{* *} P<0.01$. 


\section{SPESP1 behaves aberrantly in Eqtn ${ }^{-/-}$sperm during the acrosome reaction}

To determine how SPESP1 behaves in Eqtn ${ }^{-/}$sperm, both the staining intensity and the patterns of SPESP1 expression in Eqtn ${ }^{-/}$sperm during the acrosome reaction were compared to those in Eqtn ${ }^{++}$sperm (WT, control). In this experiment, the stage of acrosome reaction was estimated by the IZUMO1 staining pattern. As a result, SPESP1 was present in the acrosome in both Eqtn ${ }^{+/+}$ (Fig. 4A) and Eqtn ${ }^{-/-}$(Fig. 4B) sperm. SPESP1 in Eqtn ${ }^{+/+}$ sperm showed stable staining intensity with a stable distribution pattern at the equatorial segment during the acrosome reaction (Fig. 4A), and with gradually decreasing staining intensity as the acrosome reaction advanced. In contrast, SPESP1 in Eqtn ${ }^{-/-}$sperm showed variable staining intensity and distributions in the acrosome, starting from an early stage of the acrosome reaction. The results show the most representative patterns of SPESP1 expression at each stage (Fig. 4B). In detail, at an early to middle stage, SPESP1 was most frequently located at the anterior part of the equatorial segment. Later, at the middle to late stage, SPESP1 was variously distributed, showing an irregularly stained patterns at the equatorial segment. Immunostaining of
IZUMO1 was somewhat aberrant in the early to middle stage but normal in the late stage of the acrosome reaction in $\mathrm{Eqtn}^{-/-}$sperm. A comparison was made to that of $\mathrm{Eqtn}^{+/+}$sperm.

\section{Eqtn/Spesp1-/- males have severely reduced fertility}

Since SPESP1 behaved aberrantly in Eqtn ${ }^{-/-}$sperm during the acrosome reaction, we generated EQTN/ SPESP1-double KO mice, and examined how the ablation of both Eqtn and Spesp 1 genes affects fertility in Eqtn/Spesp $1^{-/-}$sperm by mating Eqtn/Spesp $1^{-/-}$ males with WT females (Fig. 5A). Eqtn/Spesp $1^{-/-}$sperm showed an abnormal IZUMO1 immunostaining patterns in the head after the acrosome reaction (Fig. 5B) as reported previously (Fujihara et al. 2010), but they were grossly normal when examined by electron microscopy (Fig. 5C). EQTN/SPESP1-double KO males have severely reduced fertility, compared to EQTN-KO and WT $\left(E q n^{+/+}\right)$males; the average number of fetuses was 3.0 (a $70 \%$ reduction), 6.3 (a $37 \%$ reduction) and 10 (regarded as $100 \%$ ), respectively (Fig. 5D). Interestingly, 20\% (4 of 20) of males in the EQTN/SPESP1-double KO mice were completely infertile, even though they were paired with WT females for up to 4 months. As a reference,
A

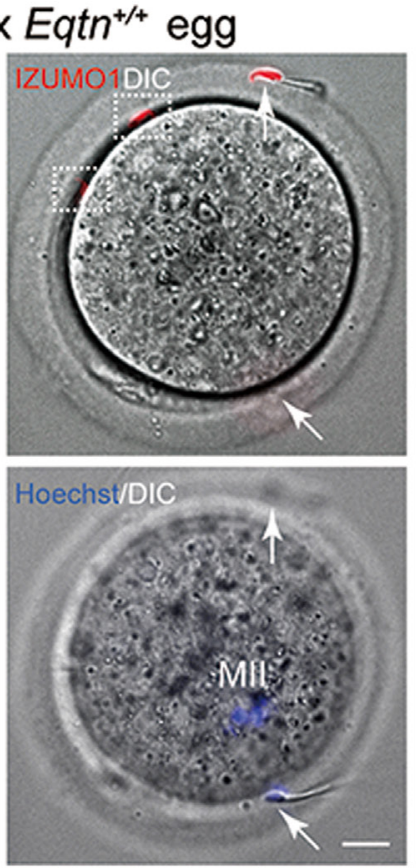

B


\section{Cd9-EGFP egg}
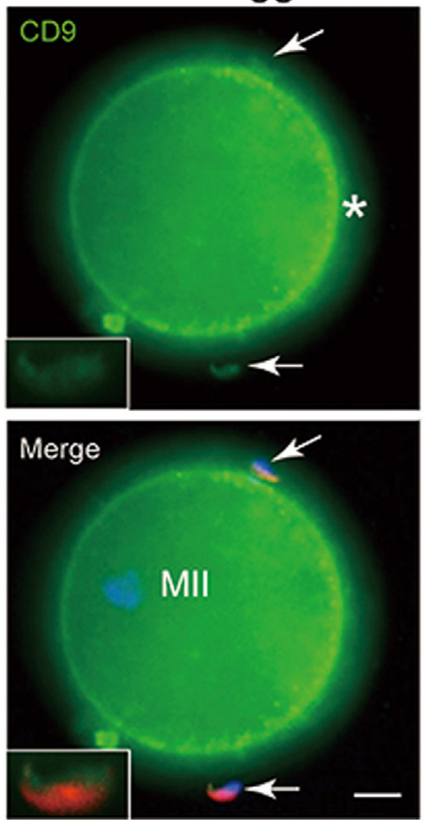

Figure 3 Presence of IZUMO1 and CD9 in Eqtn ${ }^{-/-}$sperm that reached the PVS. (A and B) IIF with anti-IZUMO1 antibody, counterstained with Hoechst. The samples were not treated with 0.1\% Triton-X 100 to permeabilize sperm membrane. Red; IZUMO1. Green; CD9. Blue (Hoechst) for sperm nuclei and egg chromosomes at MII. (A) Two Eqtn ${ }^{-/-}$sperm stained red are present in the PVS (boxes), and two redundant sperm on zona pellucida (arrows in DIC image; upper one is out of focus). Inset: Higher magnification of sperm head showing the presence of IZUMO1.

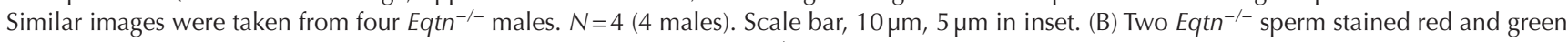
(arrows). The PVS is filled with CD9-EGFP (*, green) derived from Cd9-/--Tg(Cd9-Egfp) used in this experiment. Inset: Sperm heads stained red and green. Presence of Juno in the PVS is shown in Supplementary Fig. 5 . Similar images were taken from three Eqtn ${ }^{\prime-}$ males. $N=3$ (3 males). Scale bar, $10 \mu \mathrm{m}, 1 \mu \mathrm{m}$ in inset. ES, equatorial segment; PAR, postacrosomal region. 
A

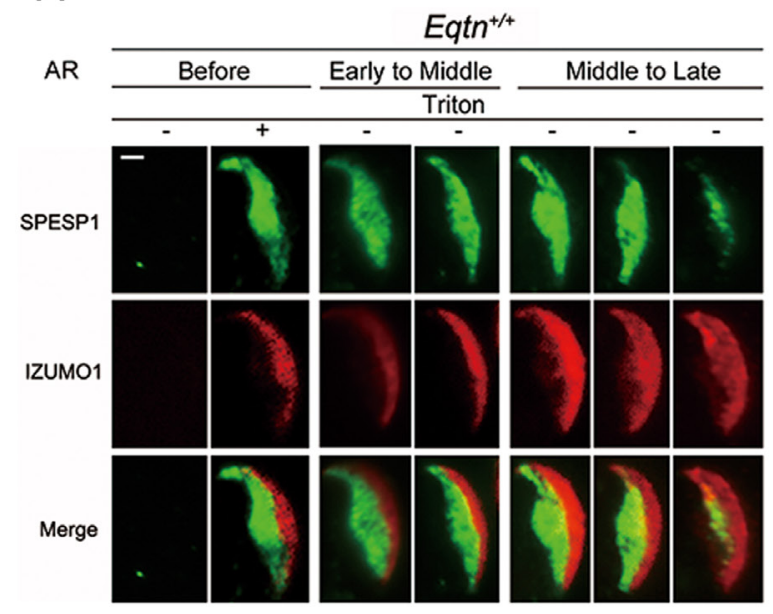

B

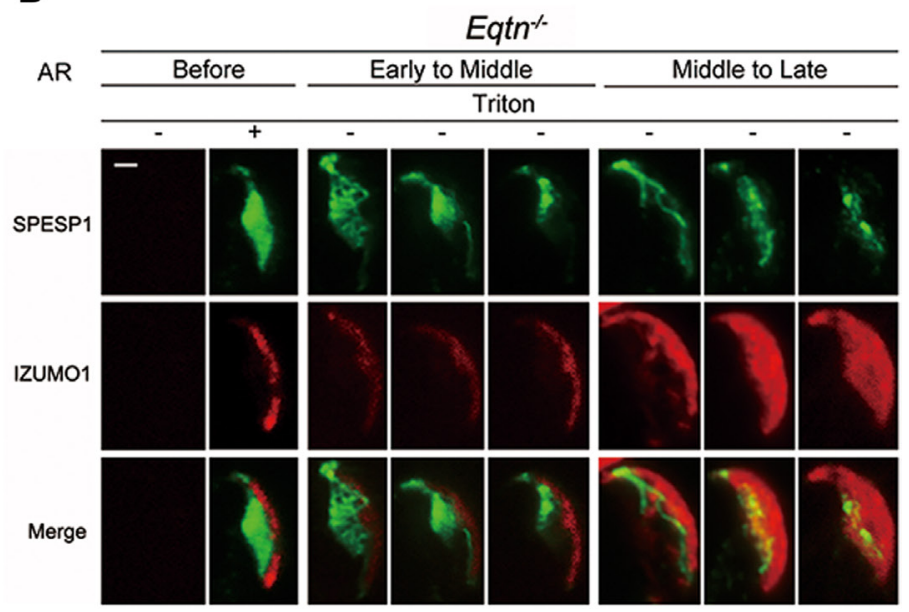

Figure 4 Aberrant behavior of SPESP1 in Eqtn ${ }^{-/-}$sperm during the acrosome reaction (AR). (A and B) IIF with anti-SPESP1 (green) and antiIZUMO1 antibody (red). Immunostaining of IZUMO1 was somewhat aberrant in the early to middle stage but normal in the late stage of the AR

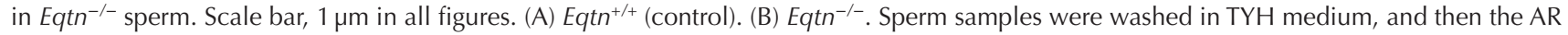
was induced with A-23187. The sperm samples were fixed with paraformaldehyde, but not treated with Triton-X-100 to permeabilize membrane. The experiments were triplicated (3 males each), in which more than 100 sperm were analyzed at each stage. Detailed characteristics are described in the text.

Spesp $1^{-1-}$ sperm reached eggs and showed partially reduced fertility, with an average litter size of 7.8 pups as reported (Fujihara et al. 2010). Eqtn/Spesp $1^{-/-}$sperm could reach the PVS of the MII egg but could not enter the cytoplasm. The Eqtn/Spesp $1^{-1-}$ sperm that reached and accumulated in the PVS also had unusual granular or clustered IZUMO1 immunostaining of IZUMO1 in the head (Fig. 5E).

\section{The defects caused by Eqtn ${ }^{-/-}$males were rescued by Eqtn $^{-/-}$-Tg(Eqtn) males}

To verify the importance of EQUATORIN, we rescued $E_{\text {Etn }}{ }^{-1-}$ males with Eqtn ${ }^{-1-}$-Tg(Eqtn) males (Fig. 6A). The generated Eqtn ${ }^{-/-} \operatorname{Tg}(E q t n)$ males were fertile, and the sperm were normal in morphology showing a typical localization pattern of EQUATORIN (more than $N=100)$ (Fig. 6B). Eqtn ${ }^{-/-}-\mathrm{Tg}(E q t n)$ males, which were mated with WT females, had almost completely restored the defects caused by Eqtn ${ }^{-/-}$males when analyzed by the pronucleus formation assay (Fig. 6C) and by fertility rate (Fig. 6D). The fertility rate was determined by the number of pups obtained.

\section{Discussion}

In this study, we have provided further evidence for the in vivo role of EQUATORIN using EQTN-KO males. The EQUATORIN-deficient sperm are normal in the morphology, they induce the acrosome reaction, they penetrate the zona pellucida and reach the PVS, but they have significantly reduced fertility. The reduction is due to sperm-egg adhesion failure as shown by fertility assays, time-lapse imaging and in vitro gamete binding assays using activated sperm and zonafree oocytes. In addition, both the Eqtn ${ }^{-/}$and Eqtn/ Spesp $1^{-1-}$ sperm that reached the PVS of MII eggs showed strong IZUMO1 immunostaining (Figs $3 \mathrm{~A}$ and $5 \mathrm{E}$, respectively). The staining pattern was normal in the $E q \mathrm{tn}^{-/-}$sperm, although it was somewhat unusual in the Eqtn/Spesp $1^{-1-}$ sperm. This result further supports the notion that the fertility reduction is due to sperm-egg adhesion failure. The ratio of EQUATORIN involvement in fertilization ranges from 24 (Fig. 2A) to 37\% (Fig. 5D). This conclusion was supported by the rescue experiment using the Eqtn ${ }^{--}-\mathrm{Tg}($ Eqtn) male, since they almost completely recovered the fertility (Fig. 6).

This study agrees with a previous study using another EQUATORIN-deficiency mutant line Eqtn ${ }^{\text {tm1Gar }}$ in terms of male subfertility (Hao et al. 2014). However, there is a difference in the failure process; during the acrosome reaction (Hao et al. 2014) versus during the sperm-egg adhesion seen in this study. Although the exact reason of the difference is uncertain, there are several differences in these two studies. First, exon 4 was deleted in different ways. We deleted exons from 1 to 5 (Supplementary Fig. 1B and C) and used Eqtn ${ }^{-/-}$males back-crossed more than ten times. In contrast, exons from 4 to 7 were deleted in the Eqtn ${ }^{\text {tmlGar }}$ mice, but it is unclear how many times the Eqtn ${ }^{\text {tm1Gar }}$ males were back-crossed. EQUATORIN protein is not produced in both Eqtn ${ }^{\text {tm1Gar }}$ and EQTN-KO mice, since similar methods using 129S/ Sv-derived embryonic stem cells and C57BL/6J mice were used to make chimeric mice. However, Eqtn ${ }^{\text {tm1Gar }}$ line was maintained by mixing $\mathrm{C} 57 \mathrm{BL} / 6 \mathrm{~J}$ and 129 background mice by the donating laboratory according 
A

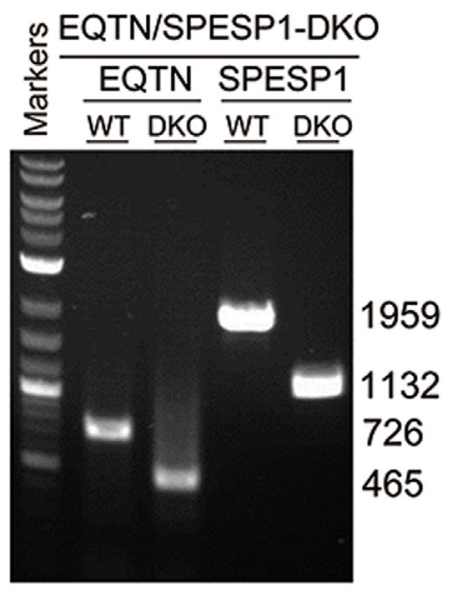

$\mathrm{D}$

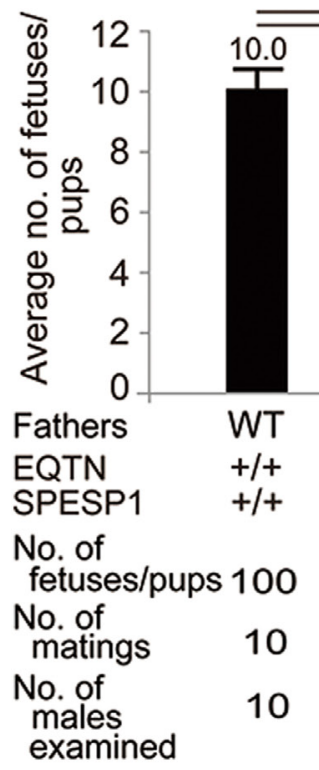

B

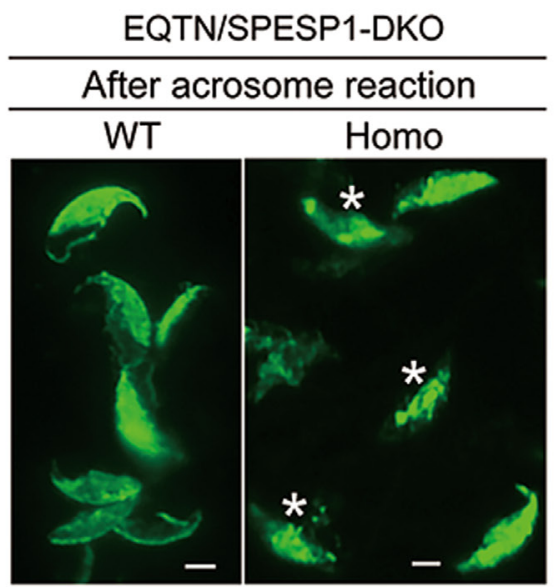

C

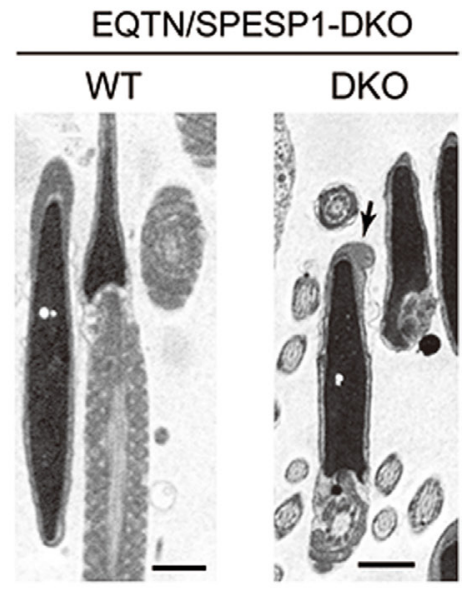

$\mathrm{E}$

Figure 5 Reduction of male fertility caused by EQTN/SPESP1-double KO (DKO) males or sperm after mating with WT females. (A) PCR showing deletions of Eqtn and Spesp1 in EQTN/SPESP1-double KO male. Numerals at the right side of the lane indicate the size of the bases. (B) IIF with anti-IZUMO1 antibody. EQTN/SPESP1-double KO sperm showed abnormal immunostaining of IZUMO1 (green) after the AR, compared to WT. Sperm were not treated with $0.1 \%$ Triton-X 100 . More than 100 sperm were examined. $N=3$ (3 males). Scale bar, $5 \mu \mathrm{m}$. (C) Transmission electron microscopy. EQTN/SPESP1-double KO sperm morphology is grossly normal, compared to WT. Small bending of the anterior acrosome region in this figure (arrow) is not significant. More than 50 sperm were examined. $N=3$ (3 males). Scale bar, $1 \mu \mathrm{m}$. (D) Average number of fetuses/pups is significantly reduced in EQTN-KO (6.3 fetuses) and EQTN/SPESP1-double KO (3.0 fetuses), compared to wild-type (WT; 10.0 pups). EQTN/SPESP1-double KO data were obtained from 44 mating trials in total using 20 males including four infertile males; the production of fetuses ranged from 0 to 6 . Bars in the graph show S.E.M. ${ }^{* *} P<0.01$. (E) Immunostaining of IZUMO1 (green) in EQTN/SPESP1-double KO sperm accumulated in the PVS of the egg recovered from the WT females after mating with EQTN/SPESP1-double KO males. IIF with antiIZUMO1 antibody shown by differential interference contrast image. Blue (Hoechst) for nucleus. Sperm were not treated with $0.1 \%$ Triton-X 100. Two sperm are present in PVS (indicated by 1 and 2), while the third one (3) is out of focus. Among them, one sperm in the rectangular area (1) is magnified to show unusual granular or cluster immunostaining $\left(^{*}\right)$ of IZUMO1 (green) on the head (inset). Similar images were taken from three $\mathrm{Eqtn}^{-/-}$males $(\mathrm{N}=3)$. ES, equatorial segment; PAR, postacrosomal region. Scale bar, $10 \mu \mathrm{m}, 5 \mu \mathrm{m}$ in inset.

to the Jackson Laboratory (Stock No. 007733), while EQTN-KO line was back-crossed more than ten times and maintained by only $\mathrm{C} 57 \mathrm{BL} / 6 \mathrm{JJmsSlc}$ mice to make the congenic line in this study. Therefore, some differences in the genetic background may account for the differences. Some caution may be needed when analyzing phenotypes of mutant animals in one genetic background. The MN9 antibody, whose epitope is present around an O-glycosylation at threonine 138 (Thr138, Supplementary Fig. 1A and B) in the exon 4, 
A
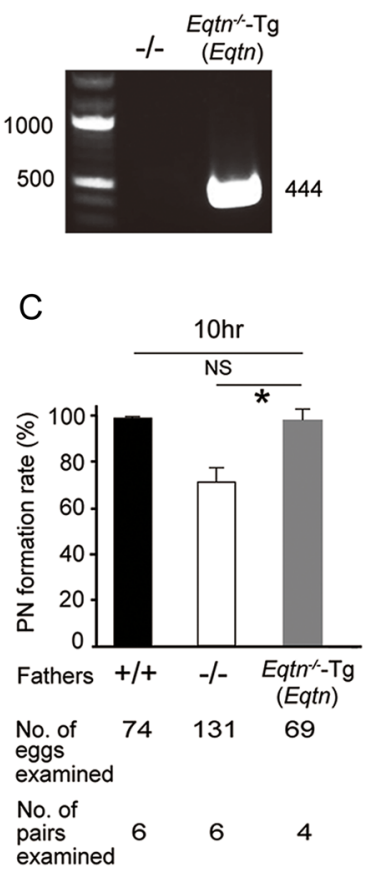

B



D

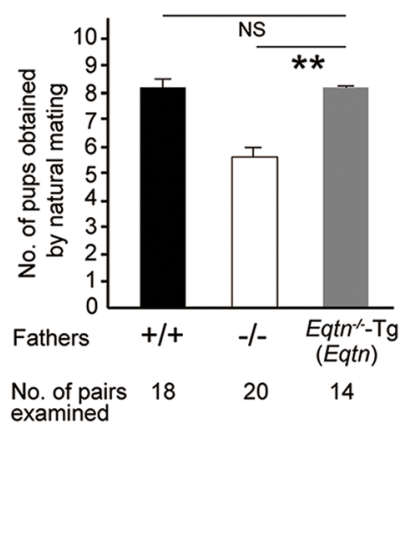

Figure 6 Rescue of Eqtn ${ }^{-/-}$male fertility reduction by Eqtn-/-$\mathrm{Tg}($ Eqtn) males after mating with wild-type females. (A) PCR results for genotyping. The absence of Equatorin (Eqtn) in Eqtn ${ }^{-/-}$males $(-/-)$ and presence of Eqtn (444) in Eqtn ${ }^{-/-}-\operatorname{Tg}($ Eqtn) males. $N=3$ (3 males). (B) Presence of EQUATORIN (red) in Eqtn ${ }^{-1-}-\mathrm{Tg}(E q t n)$ sperm shown by IIF with MN9 antibody. DIC; differential interference contrast. Sperm were treated with $0.1 \%$ Triton-X 100 to permeabilize membrane. More than 100 sperm were examined. $N=3$ (3 males).

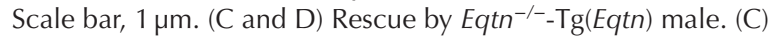
Pronucleus (PN) formation rate at $10 \mathrm{~h}$ postplug formation. (D) Number of pups obtained by natural mating. WT (+/+). Eqtn ${ }^{-/-}(-/-)$. Bars in the graph show S.E.M. ${ }^{*} P<0.05 .{ }^{* *} P<0.01$.

suppresses fertilization approximately at the sperm-egg fusion process in vitro (Toshimori et al. 1998) and in vivo (Yoshinaga et al. 2001). Thus, the results of the present study are consistent with those of our previous studies.

It is expected that EQUATORIN function is facilitated by SPESP1, since the male fertility was more severely reduced in EQTN/SPESP1-double KO males (3.0 pups) when compared to EQTN-KO (6.3 pups) in the present study and compared to SPESP1-KO males (7.8 pups) in the previous report (Fujihara et al. 2010). In addition, SPESP1 showed aberrant behavior in Eqtn ${ }^{-/-}$sperm during the acrosome reaction in the present study, while EQUATORIN showed an aberrant distribution in Spesp $1^{-1-}$ sperm in the previous report (Fujihara et al. 2010). There is a possibility that EQUATORIN stabilizes SPESP1 and the opposite is also true, by which EQUATORIN is facilitated to move to the surface of the equatorial segment during the acrosome reaction, but the detailed mechanism is unclear at this moment.

In the present study, not only IZUMO1 and CD9 in Eqtn ${ }^{-/-}$sperm but also IZUMO1 in Eqtn/Spesp 1 $1^{-/-}$

sperm were not enough to compensate for the reduced fertility caused by Eqtn ${ }^{-/}$sperm and by Eqtn/Sp esp $1^{-1-}$ sperm, respectively. Conversely, EQUATORIN was not enough to compensate for the reduced fertility caused by Izumo $1^{-1-}$ sperm, as reported previously (Inoue et al. 2005). Considering these data, it is possible to speculate whether EQUATORIN and IZUMO1 play different roles in the process from sperm-egg adhesion to fusion. EQUATORIN relocates from inside of the equatorial segment to the equatorial plasma membrane and remains in the egg (Manandhar \& Toshimori 2001), while IZUMO1 relocates from inside of the anterior acrosome to the whole head plasma membrane and does not remain in the egg (Satouh et al. 2012).

Considering the decreased body weight of fetuses in the experiment using Eqtn ${ }^{-/-}$fathers, we cannot deny the possibility that EQUATORIN may have an additional role that relates to embryonic development. Our previous study suggested that some amount of EQUATORIN remained in the fertilizing sperm (Manandhar \& Toshimori 2001). Therefore, it is possible that some amount of EQUATORIN located on the side of the inner acrosomal membrane is incorporated in eggs and facilitates early embryonic development. However, the details are unclear at this moment.

EQUATORIN is preferentially integrated into the developing spermatid acrosome as a 40-50-kDa $N$, $\mathrm{O}$-sialoglycoprotein during spermatogenesis in the testis (Supplementary Fig. 1A; Ito et al. 2013), and then the molecular size is reduced to approximately $35 \mathrm{kDa}$ in acrosome-reacted sperm (Yoshida et al. 2010). Therefore, the $\mathrm{N}$ - and $\mathrm{C}$-terminal domains may be timedependently cleaved in sperm during fertilization by a still unknown mechanism.

\section{Supplementary data}

This is linked to the online version of the paper at https://doi.org/10.1530/REP-18-0394.

\section{Declaration of interest}

The authors declare that there is no conflict of interest that could be perceived as prejudicing the impartiality of the research reported.

\section{Funding}

This work was supported by Grant-in-Aid from the Ministry of Education, Culture, Sports, Science and Technology of Japan (MEXT) (grant numbers No. 22112504, No. 22390033, No. 24112706, No. 25293041) to K T, (grant number No. 24592441) to C I, and (grant number No. 26861311) to $\mathrm{K}$ $\mathrm{Y}$, and in part by CREST/JST of Japan (grant number No. 24592441) to KT. 


\section{Acknowledgements}

The authors thank Drs J Takeda and T Ijiri for providing the targeting vector, Drs M Okabe and M Ikawa for providing the antibodies and our colleagues T Mutoh, K Kamimura, Y Tajima, $\mathrm{K}$ Ushikoshi and T Kanamori for their technical support. They thank AJE (www.aje.com/jp) for English language editing.

\section{References}

Bianchi E, Doe B, Goulding D \& Wright GJ 2014 Juno is the egg Izumo receptor and is essential for mammalian fertilization. Nature $\mathbf{5 0 8}$ 483-487. (https://doi.org/ 10.1038/nature13203)

Evans JP 2012 Sperm-egg interaction. Annual Review of Physiology 74 477-502. (https://doi.org/10.1146/annurev-physiol-020911-153339)

Florman HM \& Fissore RA 2014 Fertilization in Mammals. In The Physiology of Reproduction, edn 4, pp 149-196. Eds TM Plant and AJ Zeleznik. London: Academic Press.

Fujihara Y, Murakami M, Inoue N, Satouh Y, Kaseda K, Ikawa M \& Okabe M 2010 Sperm equatorial segment protein 1, SPESP1, is required for fully fertile sperm in mouse. Journal of Cell Science 123 1531-1536. (https://doi.org/10.1242/jcs.067363)

Hao J, Chen M, Ji S, Wang X, Wang Y, Huang X, Yang L, Wang Y, Cui X \& Lv L et al. 2014 Equatorin is not essential for acrosome biogenesis but is required for the acrosome reaction. Biochemical and Biophysical Research Communications 444 537-542. (https://doi.org/ 10.1016/j. bbrc.2014.01.080)

Inoue N, Ikawa M, Isotani A \& Okabe M 2005 The immunoglobulin superfamily protein Izumo is required for sperm to fuse with eggs. Nature 434 234-238. (https://doi.org/ 10.1038/nature03362)

Inoue N, Hagihara Y, Wright D, Suzuki T \& Wada I 2015 Oocyte-triggered dimerization of sperm IZUMO1 promotes sperm-egg fusion in mice. Nature Communication 6 8858. (https://doi.org/ 10.1038/ncomms9858)

Ito C, Yamatoya K, Yoshida K, Kyono K, Yao R, Noda T \& Toshimori K 2010 Appearance of an oocyte activation-related substance during spermatogenesis in mice and humans. Human Reproduction $\mathbf{2 5}$ 2734-2744. (https://doi.org/10.1093/humrep/deq216)

Ito C, Yamatoya K, Yoshida K, Fujimura L, Hatano M, Miyado K \& Toshimori K 2013 Integration of the mouse sperm fertilization-related protein equatorin into the acrosome during spermatogenesis as revealed by super-resolution and immunoelectron microscopy. Cell and Tissue Research 352 739-750. (https://doi.org/ 10.1007/s00441-013-1605-y)

Ito C, Yamatoya K \& Toshimori K 2015 Analysis of the complexity of the sperm acrosomal membrane by super-resolution stimulated emission depletion microscopy compared with transmission electron microscopy. Microscopy 64 279-284. (https://doi.org/ 10.1093/jmicro/dfu101)

Kaji K, Oda S, Shikano T, Ohnuki T, Uematsu Y, Sakagami J, Tada N, Miyazaki S \& Kudo A 2000 The gamete fusion process is defective in eggs of Cd9-deficient mice. Nature Genetics 24 279-282. (https://doi. org/ 10.1038/73502)

Le Naour F, Rubinstein E, Jasmin C, Prenant M \& Boucheix C 2000 Severely reduced female fertility in CD9-deficient mice. Science $\mathbf{2 8 7}$ 319-321. (https://doi.org/ 10.1126/science.287.5451.319)

Li YC, Hu XQ, Zhang KY, Guo J, Hu ZY, Tao SX, Xiao LJ, Wang QZ, Han CS \& Liu YX 2006 Afaf, a novel vesicle membrane protein, is related to acrosome formation in murine testis. FEBS Letters $\mathbf{5 8 0}$ 4266-4273. (https://doi.org/ 10.1016/j.febslet.2006.06.010)

Lorenzetti D, Poirier C, Zhao M, Overbeek PA, Harrison W \& Bishop CE 2014 A transgenic insertion on mouse chromosome 17 inactivates a novel immunoglobulin superfamily gene potentially involved in spermegg fusion. Mammalian Genome 25 141-148. (https://doi.org/ 10.1007/ s00335-013-9491-x)

Miyado K, Yamada G, Yamada S, Hasuwa H, Nakamura Y, Ryu F, Suzuki K, Kosai K, Inoue K \& Ogura A et al. 2000 Requirement of CD9 on the egg plasma membrane for fertilization. Science 287 321-324. (https://doi. org/ 10.1126/science.287.5451.321)

Miyado K, Yoshida K, Yamagata K, Sakakibara K, Okabe M, Wang X, Miyamoto K, Akutsu H, Kondo T \& Takahashi Y et al. 2008 The fusing ability of sperm is bestowed by CD9-containing vesicles released from eggs in mice. PNAS 105 12921-12926. (https://doi.org/10.1073/ pnas.0710608105)
Manandhar G \& Toshimori K 2001 Exposure of sperm head equatorin after acrosome reaction and its fate after fertilization in mice. Biology of Reproduction 65 1425-1436. (https://doi.org/10.1095/ biolreprod65.5.1425)

Nakanishi T, Ikawa M, Yamada S, Parvinen M, Baba T, Nishimune Y \& Okabe M 1999 Real-time observation of acrosomal dispersal from mouse sperm using GFP as a marker protein. FEBS Letters 449 277-283. (https://doi.org/10.1016/S0014-5793(99)00433-0)

Okabe M 2013 The cell biology of mammalian fertilization. Development 140 4471-4479. (https://doi.org/ 10.1242/dev.090613)

Primakoff P \& Myles DG 2007 Cell-cell membrane fusion during mammalian fertilization. FEBS Letters 581 2174-2180. (https://doi.org/ 10.1016/j.febslet.2007.02.021)

Ruiz A, Pujana MA \& Estivill X 2000 Isolation and characterisation of a novel human gene (C9orf11) on chromosome 9p21, a region frequently deleted in human cancer. Biochimica et Biophysica Acta 1517 128-134. (https://doi.org/10.1016/S0167-4781(00)00272-4)

Satouh Y, Inoue N, Ikawa M \& Okabe M 2012 Visualization of the moment of mouse sperm-egg fusion and dynamic localization of IZUMO1. Journal of Cell Science 125 4985-4990. (https://doi.org/ 10.1242/ jcs.100867)

Toshimori K \& Eddy EM 2014 The spermatozoon. In The Physiology of Reproduction, edn 4, pp 99-148. Eds TM Plant and AJ Zeleznik. London: Academic Press.

Toshimori K, Saxena DK, Tanii I \& Yoshinaga K 1998 An MN9 antigenic molecule, equatorin, is required for successful sperm-oocyte fusion in mice. Biology of Reproduction 59 22-29. (https://doi.org/10.1095/ biolreprod59.1.22)

Toshimori K, Tanii I, Araki S \& Oura C 1992 Characterization of the antigen recognized by a monoclonal antibody MN9: unique transport pathway to the equatorial segment of sperm head during spermiogenesis. Cell and Tissue Research 270 459-468. (https://doi. org/10.1007/BF00645047)

Toyoda Y, Yokoyama M \& Hoshi T 1971 Studies on fertilization of mouse eggs in vitro: I. In vitro fertilization of eggs by fresh epididymal sperm. Japanese Journal of Animal Reproduction 16 147-151. (https://doi. org/10.1262/jrd1955.16.152)

Wang H, Luo J, Carlton C, McGinnis LK \& Kinsey WH. 2017 Spermoocyte contact induces outside-in signaling via PYK2 activation. Developmental Biology $\mathbf{4 2 8}$ 52-62. (https://doi.org/ 10.1016/j. ydbio.2017.05.016)

Wassarman PM 2014 Reproductive biology: sperm protein finds its mate. Nature 508 466-467. (https://doi.org/ 10.1038/nature13227)

Wolkowicz MJ, Digilio L, Klotz K, Shetty J, Flickinger CJ \& Herr JC 2008 Equatorial segment protein (ESP) is a human alloantigen involved in sperm-egg binding and fusion. Journal of Andrology 29 272-282. (https://doi.org/ 10.2164/jandrol.106.000604)

Yamatoya K, Yoshida K, Ito C, Maekawa M, Yanagida M, Takamori K, Ogawa H, Araki Y, Miyado K \& Toyama Y et al. 2009 Equatorin: identification and characterization of the epitope of the MN9 antibody in the mouse. Biology of Reproduction 81 889-897. (https://doi. org/10.1095/biolreprod.109.077438)

Yanagimachi R 1994 Mammalian fertilization. In The Physiology of Reproduction, edn 2, pp 189-317. Eds E Knobil \& JD Neill. New York: Raven Press.

Yoshida K Ito C, Yamatoya K, Maekawa M, Toyama Y, Suzuki-Toyota F \& Toshimori K. 2010 A model of the acrosome reaction progression via the acrosomal membrane-anchored protein equatorin. Reproduction 139 533-544. (https://doi.org/10.1530/REP-09-0434)

Yoshinaga K, Saxena DK, Oh-oka T, Tanii I \& Toshimori K 2001 Inhibition of mouse fertilization in vivo by intra-oviductal injection of an antiequatorin monoclonal antibody. Reproduction 122 649-655. (https:// doi.org/10.1530/rep.0.1220649)

Received 30 July 2018

First decision 31 August 2018

Revised manuscript received 21 September 2018

Accepted 1 October 2018 